\title{
Framework of Automatic Robot Surgery System using Visual Servoing
}

\author{
Takayuki Osa, Christoph Staub and Alois Knoll
}

\begin{abstract}
The use of technology for automation of surgical tasks has great potential in the field of robotic surgery. Automation of surgical robots essentially involves designing a control scheme for precise and automatic positioning of robotic manipulators. This objective can be achieved by the use of Visual servoing.

We have developed a visual-servoing-based robotic laparoscopic surgery system. The visual servoing system enables autonomous control of a robot arm in a surgical set-up. In this paper, we propose algorithms for the automatic positioning of the laparoscope and the surgical instruments under small incisions of skin. We have verified the performance of the system theoretically and experimentally.
\end{abstract}

\section{INTRODUCTION}

Robotic systems for minimally invasive surgery (MIS) have undergone considerable improvement in recent years[10]. MIS has the potential to reduce scarring and shorten recovery times.

Despite this progress in medical robots, MIS is still timeconsuming and leads to quicker fatigue in surgeons. Hence a lot of researchers are working on automating surgical tasks. To achieve this goal, it is important to establish a control scheme for robotic surgery systems.

Controlling robots based on feedback from computer vision is one way to achieve this objective. This type of control is called visual servoing. Visual servoing has the potential to control the robots adaptively in response to the environment. D. Kragic et al provide a good framework for visual servoing [8] [9]. F. Chaumette and S. Hutchinson developed a visual servoing tutorial[1][2].

Some robotic surgery systems using the visual servoing system have already been proposed.

R. Ginhoux et al. have developed a robotic surgery system, which can track the physiological motion of organs [3][4]. They have proposed a predictive control scheme to track the physiological motion and control the system using visual servoing. However, constraint of surgical instruments in MIS is not considered in this paper. P. Hynes et al. developed a robotic surgery system using visual servoing and conducted autonomous suturing [6][7]. Their system is able to position

This work was supported by the German Research Foundation (DFG) within the Collaborative Research Center SFB 453 on "High-Fidelity Telepresence and Teleaction".

T. Osa is with Department of Engineering Synthesis, University of Tokyo, 7-3-1 Hongo Bunkyoku Tokyo, 113-8656, Japan osa@nml.t.u-tokyo.ac.jp

C. Staub is with the Department of Informatics,Technical University Munich, Boltzmannstrasse 3, Garching bei Munich, 85748, Germany staublin.tum.de

A. Knoll is with the Department of Informatics,Technical University Munich, Boltzmannstrasse 3, Garching bei Munich, 85748, Germany knollein.tum.de the instrument and they performed suturing by setting the desired points manually. However, performance parameters of the system, such as positioning precision of the instruments, were not analyzed.

We have developed a robotic surgery system with a laparoscope and instruments for MIS. The laparoscope and surgical instruments are mounted on robot arms and these devices can be positioned automatically using visual servoing. We have proposed algorithms to control the laparoscope and the instruments beneath small incisions of the skin. To control the laparoscope in a small incision, we modified the interaction matrix for image-based visual servoing. In addition, we have verified the performance of the system theoretically and experimentally. To estimate the performance of the system, we propose to analyze the configuration of the stereo camera and the condition number of the interaction matrix of visual servoing.

The composition of this paper is as follows: in section II, we describe the system setup. The control scheme is described in section III, where the constraint of keeping the insertion points on the skin is introduced, and the interaction matrices of our system are mentioned. In section IV, we present an analysis of our system. The range of the system workspace is estimated by analyzing the system configuration. In addition, the condition number of the interaction matrix is considered to investigate the robustness of the system.

In section VI, experiments to evaluate the system performance are described. The surgical robot system showed satisfactory performance. Conclusions and future works are presented in the final section.

\section{SYSTEM SETUP}

Our robotic surgery system is based on the robotic system for laparoscopic surgery, which has already been reported in [11][12]. The experimental system setup is shown in Fig.1. Four robot arms (Mitsubishi MELFA 6SL, Mitsubishi Electric Corp.) are mounted on the ceiling such that they do not block surgeons from accessing the operation table. Surgical instruments are attached to three robot arms. A laparoscope is attached to the fourth arm; the laparoscope provides surgeons with stereo images during surgical procedures. The laparoscope has lenses with strong distortion to provide the surgeons with a wide view.

In our system, hand-eye calibration and camera calibration were performed and the relative positions and orientation between each robot arm and the laparoscope were approximated. These calibrations enable the real-time computation of the relative positions and orientations of each instrument 
and the laparoscope. The instruments and surgical needles are tracked by color segmentation. Colored markers are attached on the shaft of the instrument, and the position of the tip of the instrument is estimated from the position of these markers.

\section{CONTROL SCHEME}

For this system, image-based visual servoing is applied (see e.g. [2]). Position-based visual servoing (PBVS) is highly dependent on the accuracy of system calibrations, such as camera calibration or hand-eye calibration. On the contrary, image-based visual servoing (IBVS) is robust to calibration errors. This feature is very suitable for laparoscopic surgery.

In surgical operations, laparoscopes which have lenses with high distortion are used to provide a wide view to the surgeons. It can cause relatively high calibration error in camera parameters. The robustness of the closed loop control makes it possible to position the robots very precisely even with coarse system calibrations, using IBVS. For this reason, IBVS is applied to control the linear velocity of the robot arm. At the same time, we employed a geometric constraint, considering insertion points of the surgical instruments. It reduces the dimensionality in the interaction matrix and determines the angular velocity of the robot arms. In instrument control, stereo vision is employed to achieve highly precise positioning. Meanwhile, monocular vision is employed in laparoscope control since it is not necessary to control the laparoscope along the depth of insertion.

\section{A. Image-based visual servoing}

The control law in IBVS is described as follows

$$
\xi=-\lambda \widehat{L}^{+} e
$$

where $\xi$ is the velocity of the camera in Cartesian coordinates, $\widehat{L}^{+}$is Moore-Penrose pseudoinverse of the estimated interaction matrix, and $e$ is the error between the desired position and the current position of feature points [1].

The movement in the normalized image coordinates $\dot{s}=$ $(\dot{u}, \dot{v})^{T}$ and the velocity in Cartesian coordinates $\xi$ is related as follows.

$$
\dot{s}=L \xi
$$

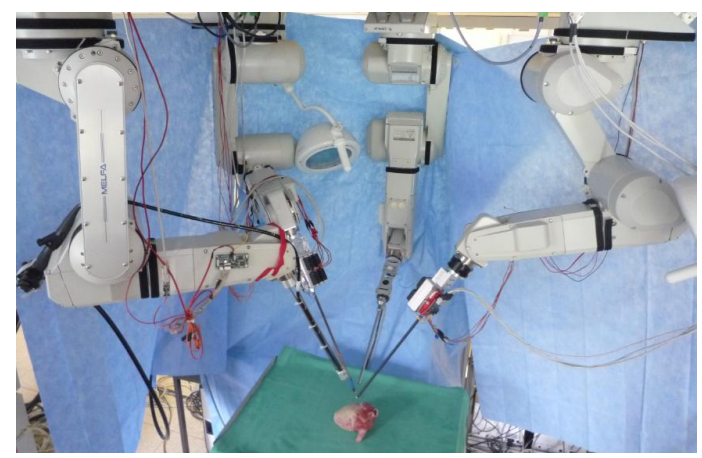

Fig. 1. System setup: three surgical instruments and a laparoscope are attached to robot arms where

$$
L=\left[\begin{array}{cccccc}
-\frac{1}{Z} & 0 & \frac{u}{Z} & u v & -1-u^{2} & v \\
0 & -\frac{1}{Z} & \frac{v}{Z} & 1+v^{2} & -u v & -u
\end{array}\right]
$$

In (3), variable $Z$ is the distance between the feature points and the origin of camera coordinates. Obviously, $Z$ has to be estimated in image-based visual servoing. In our system, every device is calibrated geometrically and the relative position and orientation between each robot arm, the laparoscope and the instruments can be computed. To compute $Z$ online, forward kinematics of each robot arm is solved and the distance between the laparoscope and the tip of the instrument is calculated.

In order to compute the normalized image coordinates $s=(u, v)^{T}$, it is necessary to calibrate the camera. Unfortunately, it is difficult to calibrate cameras which have heavy distorted images, such as the one utilized in our system. However, calibration errors do not seriously affect the system performance due to the robustness of the imagedbased closed control loop.

\section{B. Trocar Constraint}

In laparoscopic surgical operations, all instruments are inserted through trocars on the skin. It is important that the instrument does not move at the insertion point. This means that two degrees of freedom of the robot arm is determined when the position of the insertion point and the linear velocity of the instrument are known.

This constraint can be described in the following way: The velocity of the insertion point $\xi_{p}^{p}=\left(v_{p}^{p}, \omega_{p}^{p}\right)^{T}$ and the velocity of the tip of the instrument $\xi_{t}^{t}=\left(v_{t}^{t}, \omega_{t}^{t}\right)^{T}$ are related as follows

$$
\begin{aligned}
V_{t}^{p} \xi_{t}^{t} & =\xi_{p}^{p} \\
{\left[\begin{array}{cc}
R_{t}^{p} & S\left(t_{t}^{p}\right) R_{t}^{p} \\
\mathbf{0} & R_{t}^{p}
\end{array}\right]\left[\begin{array}{c}
v_{t}^{t} \\
\omega_{t}^{t}
\end{array}\right] } & =\left[\begin{array}{c}
v_{p}^{p} \\
\omega_{p}^{p}
\end{array}\right]
\end{aligned}
$$

where $\left(R_{t}^{p}, t_{t}^{p}\right)$ is transformation from the insertion point to the tip of the instrument and $S\left(t_{t}^{p}\right)$ is the skew symmetric matrix associated with the vector $t_{t}^{p}$.

Assuming that the instrument is straight, $R_{t}^{p}$ is an identity matrix and $t_{t}^{p}=(0,0, d)^{T}$, where $d$ is the distance from the insertion point to the tip of the instrument. The linear velocity at the insertion point is $v_{p}^{p}=\left(0,0, v_{p, z}^{p}\right)$.

Thus, the angular velocity at the tip of the instrument $\omega_{t}^{t}=$ $\left(\omega_{t, x}^{t}, \omega_{t, y}^{t}, \omega_{t, z}^{t}\right)^{T}$ and the linear velocity at the tip of the instrument $v_{t}^{t}=\left(v_{t, x}^{t}, v_{t, y}^{t}, v_{t, z}^{t}\right)^{T}$ are related as

$$
\left[\begin{array}{c}
0 \\
0 \\
v_{p, z}^{p}
\end{array}\right]=\left[\begin{array}{c}
v_{t, x}^{t}-d \omega_{t, y}^{t} \\
v_{t, y}^{t}+d \omega_{t, x}^{t} \\
v_{t, z}^{t}
\end{array}\right]
$$

Therefore, the following equation should be satisfied.

$$
\left[\begin{array}{l}
\omega_{t, x}^{t} \\
\omega_{t, y}^{t}
\end{array}\right]=\left[\begin{array}{c}
-\frac{v_{t, y}^{t}}{d} \\
\frac{v_{t, x}^{t}}{d}
\end{array}\right]
$$

The position of the insertion point is defined in Cartesian coordinates and the insertion depths of the instrument and the laparoscope can be computed using forward kinematics. 


\section{Laparoscope Control}

In the presence of the trocar constraint, we need to modify the interaction matrix to control the camera since the linear velocity and the angular velocity are not independent from each other. The interaction matrix can be rewritten in the following way, using (6).

$$
\begin{aligned}
\dot{s}= & {\left[\begin{array}{ll}
L_{v} & L_{\omega}
\end{array}\right]\left[\begin{array}{c}
v \\
\omega
\end{array}\right] } \\
= & L_{v} v_{c}^{c}+L_{\omega} \omega_{c}^{c} \\
= & {\left[\begin{array}{cc}
-\frac{1}{Z} & 0 \\
0 & -\frac{1}{Z}
\end{array}\right]\left[\begin{array}{c}
v_{x} \\
v_{y}
\end{array}\right] } \\
& +\left[\begin{array}{cc}
u v & -\left(1+u^{2}\right) \\
1+v^{2} & -u v
\end{array}\right]\left[\begin{array}{c}
-\frac{v_{y}}{d} \\
\frac{v_{x}}{d}
\end{array}\right] \\
= & {\left[\begin{array}{cc}
-\frac{1}{Z}-\frac{1}{d}\left(1+u^{2}\right) & -\frac{1}{d} u v \\
-\frac{1}{d} u v & -\frac{1}{Z}-\frac{d}{d}\left(1+v^{2}\right)
\end{array}\right]\left[\begin{array}{c}
v_{x} \\
v_{y}
\end{array}\right] }
\end{aligned}
$$

The modification yields to a new interaction matrix $L_{v, c a m}$ :

$$
L_{v, \text { cam }}=\left[\begin{array}{cc}
-\frac{1}{Z}-\frac{1}{d}\left(1+u^{2}\right) & -\frac{1}{d} u v \\
-\frac{1}{d} u v & -\frac{1}{Z}-\frac{1}{d}\left(1+v^{2}\right)
\end{array}\right]
$$

The control law can be derived as follows.

$$
v_{c}^{c}=-\lambda \widehat{L}_{v, c a m}^{-1} e
$$

From (9) and (6), the linear velocity and the angular velocity of the camera can be derived.

\section{Instrument Control}

In this section, we will introduce a scheme to control the instrument. Our system performs Point-to-Point positioning for the instrument control [5]. Therefore, 5 DOFs are necessary, $v_{x}, v_{y}, v_{z}, \omega_{x}, \omega_{y}$. The linear velocity of the instrument is derived from visual servoing and the angular velocity is determined by the trocar constraint (6). The interaction matrix for stereo vision is derived in the following way.

Tracking the image features in both stereo-camera images, if we use $\left(\dot{u}_{l}, \dot{v}_{l}\right)^{T}$ and $\left(\dot{u}_{r}, \dot{v}_{r}\right)^{T}$ as the movement in the left and right image coordinates, (2) can be rewritten as follows [1]

$$
\left[\begin{array}{c}
\dot{u}_{l} \\
\dot{v}_{l} \\
\dot{u}_{r} \\
\dot{v}_{r}
\end{array}\right]=\left[\begin{array}{c}
L_{l} \\
L_{r} V_{l}^{r}
\end{array}\right] \xi^{l}
$$

$L_{l}$ and $L_{r}$ are the interaction matrices corresponding to the left and right images, and $\xi$ is the movement of the feature point in Cartesian coordinates. $V_{l}^{r}$ is given by

$$
V_{l}^{r}=\left[\begin{array}{cc}
R_{l}^{r} & S\left(t_{l}^{r}\right) R_{l}^{r} \\
0 & R_{l}^{r}
\end{array}\right]
$$

where $\left(R_{l}^{r}, t_{l}^{r}\right)$ is the transformation from the right camera coordinates to the left camera coordinates and $S\left(t_{l}^{r}\right)$ is the skew symmetric matrix associated with the vector $t_{l}^{r}$. In this case, the interaction matrix is described as

$$
L=\left[\begin{array}{c}
L_{l} \\
L_{r} V_{l}^{r}
\end{array}\right]
$$

In our case, one point at the tip of the instrument is tracked as a feature point and used for visual servoing. It means that the angular velocity of this point is not related to the motion of the feature point in the image coordinates. Thus, only the linear velocity of the feature point needs to be considered. Therefore, (2) is rewritten as follows in this case:

$$
\dot{s}=L_{v, \text { inst }} v_{\text {inst }}^{c}
$$

where $L_{v, \text { inst }}$ is the left half of $L$, which is corresponding to the linear velocity and is derived from (12). Thus, the control law for the instrument can be written as follows

$$
v_{\text {inst }}^{c}=-\lambda \widehat{L}_{v, \text { inst }}^{+} e
$$

where $v_{\text {inst }}^{c}$ is the velocity of the instrument in camera coordinates and $e$ is the error between the current position and the desired position in the image coordinates.

In this configuration, $L_{v \text {,inst }}$ is a $4 \times 3$ matrix. Other researchers have proposed different control schemes. One of them is to use just 3 rows of the interaction matrix [7][9]. In comparison, their control law allows them to get the interaction matrix as the full-rank $3 \times 3$ square matrix. In this case, the interaction matrix is invertible, and it is not necessary to analyze the nullspace of the interaction matrix. However, this is equivalent to discarding a part of the information of the error function and the control scheme is more sensitive than ours to the error in the estimation of the error function. The accuracy of pointing can be coarse even if the control loop itself is stable.

In a surgical scenario, it is more difficult to detect the desired position and track the instrument precisely than in other conditions, due to the influence of the body liquid and specular reflections. The effect of the tracking error is critical. Therefore, the control scheme should be robust over the tracking error. For this reason, we have used the interaction matrix as a $4 \times 3$ matrix.

\section{E. Adaptive Gain}

For reasons of safety, the velocity of the controlled robot should not exceed a certain speed-limit. For this purpose, we have applied adaptive gain $\lambda$,which is described as follows.

$$
\lambda=\left\{\begin{array}{cc}
\frac{1}{\left\|\widehat{L}^{+} e\right\|^{\lambda_{0}}} & \left(\left\|\widehat{L}^{+} e\right\|<a\right) \\
\lambda_{0} & \left(\left\|\widehat{L}^{+} e\right\|>a\right)
\end{array}\right.
$$

where $\lambda_{0}$ is a constant coefficient and $a$ is a threshold to switch the control.

In this control, the velocity of the robot arm is set at a constant value in case that the norm of the error function is large. Otherwise, adaptive gain $\lambda$ is set constant and the error function will converge to 0 exponentially.

\section{ANALYSIS OF THE PERFORMANCE OF THE SYSTEM}

\section{A. Stability of Camera Control}

Let the candidate's Lyapunov function be $\mathcal{L}=\frac{1}{2}\|e\|^{2}$, we have [1]:

$$
\begin{aligned}
\dot{\mathcal{L}} & =e^{T} \dot{e} \\
& =-\lambda e^{T} L \widehat{L^{+}} e
\end{aligned}
$$


where $L$ is the interaction matrix. To show the global asymptotic stability, we need to show that

$$
\widehat{L L^{+}}>0
$$

In camera control, the interaction matrix $L_{v, \text { cam }}$ is described as (8). Its determinant and trace is

$$
\begin{gathered}
\operatorname{det} L_{v, \text { cam }}=\frac{1}{z^{2}}+\frac{1}{z d}\left(2+u^{2}+v^{2}\right)+\frac{1}{d^{2}}\left(1+u^{2}+v^{2}\right) \\
\operatorname{trace} L_{v, \text { cam }}=-\frac{2}{z}-\frac{1}{d}\left(2+u^{2}+v^{2}\right)-\frac{1}{d^{2}}
\end{gathered}
$$

Obviously, $\operatorname{det} L_{v, \text { cam }}>0$ and $\operatorname{trace} L_{v, \text { cam }}<0$ is always satisfied, since $z$ and $d$ are always positive when the feature point is in camera images. It means that $L_{v, c a m}$ is always invertible and negative-definite and (17) is satisfied. Thus, this control of the camera shows global asymptotic stability.

\section{B. Condition number}

The control law, (14) is equivalent to solving the following equation based on the least mean square method.

$$
e=L_{v, \text { inst }} v_{\text {inst }}^{c}
$$

where $e$ is the measured error in the image coordinates. In actual application, $e$ should include the measurement error $\Delta e$. Thus, (20) can be rewritten as follows:

$$
e_{\text {true }}+\Delta e=L_{v, \text { inst }}\left(v_{\text {true }}+\Delta v\right)
$$

where $e=e_{\text {true }}+\Delta e$, and $v_{\text {inst }}^{c}=v_{\text {true }}+\Delta v$. Then, $\Delta e$ and $\Delta v$ are related as follows:

$$
\frac{\|\Delta v\|}{\|v\|} \leq\left\|L_{v, \text { inst }}^{+}\right\|\left\|L_{v, \text { inst }}\right\| \frac{\|\Delta e\|}{\|e\|}
$$

where, $\|A\|$ is the maximum singular value of the matrix $A$. The number $k=\left\|L_{v, \text { inst }}^{+}\right\|\left\|L_{v, \text { inst }}\right\|$ is known as the condition number of the numerical equation. The condition number shows how robust the solution of the equation is against the measurement error. In other words, if $k$ is larger, the control law is less robust against measurement errors in the image coordinates. To choose the proper interaction matrix, the condition number should be taken into account. To compare the robustness of the control schemes, the evolution of the condition number was simulated. One of the results was as shown in Fig. 2.

The result in Fig.2 shows that the condition number in the case where 4 rows of $L_{v, \text { inst }}$ were used was smaller than the one in the case where 3 rows of $L_{v \text {,inst }}$ were used. This implies that the control scheme which uses 4 rows of $L_{v \text {,inst }}$ is more robust than the other one against the measurement error.

\section{Estimation of the Range of Workspace}

In image-based visual servoing, the control is based on the error measured in image coordinates. It means that the precision in positioning the instruments in Cartesian coordinates is dependent on the configuration of the camera, which provides the visual feedback to the control system.
In whichever system we use, there is an error in the tracking of the instrument and setting of the desired point. Those errors lead to an error between the final position of the instrument and the desired position of the instrument. This error between the final position and the desired position which is measured in image coordinates is nearly independent of the distance between the camera and the object.

From the maximum acceptable positioning error in Cartesian coordinates and the final error measured in image coordinates, we can estimate the correct range of the distance between the camera and the object.

Let us define the position of the desired point in left and right normalized image coordinates with $s_{l}=\left(u_{l}, v_{l}\right)$ and $s_{r}=\left(u_{r}, v_{r}\right)$, and the position of the desired point in Cartesian coordinates in left and right camera coordinates with $p_{l}=\left(x_{l}, y_{l}, z_{l}\right)^{T}, p_{r}=\left(x_{r}, y_{r}, z_{r}\right)^{T}$. In case of a parallel-aligned stereo camera, $s_{l}, s_{r}, p_{l}, p_{r}$ are related as follows:

$$
u_{r}-u_{l}=\frac{x_{l}-x_{r}}{z}=\frac{d}{z}
$$

where, $z_{l}=z_{r}=z$ and $d=x_{l}-x_{r}$. (23) can be rewritten as

$$
z=\frac{d}{u_{l}-u_{r}}=\frac{d}{u_{d}}
$$

where $u_{d}=u_{l}-u_{r}$. If we apply Taylor expansion to (24), we get

$$
z+\Delta z=\frac{d}{u_{d}}-\frac{d}{\left(u_{d}\right)^{2}} \Delta u+\frac{d}{\left(u_{d}\right)^{3}}(\Delta u)^{2}-\cdots
$$

If we ignore the 2 nd term, we get the relationship between $\Delta z$ and $\Delta u$ :

$$
|\Delta z|=\left|\frac{d}{z^{2}} \Delta u\right|
$$

In our systems, the final errors measured in image coordinates are within \pm 3 [pixel], which is equivalent to $|\Delta u| \leq$ 0.01. To satisfy the required precision of the system, $\Delta z$ should be less than $1 \mathrm{~mm}$. From this information and (26), the proper range of workspace is less than $80 \mathrm{~mm}$ from the laparoscope.

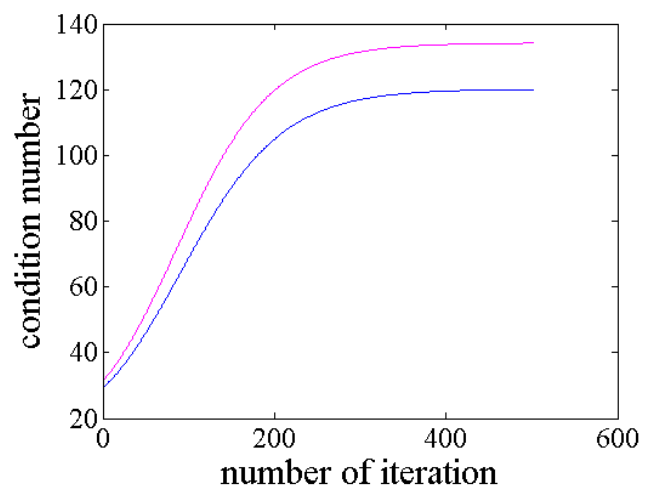

Fig. 2. The lines shows the change of condition numbers. The red line shows the result in case that 3 rows of $L_{v}$,inst were used, and the blue line shows the result in case that 4 rows of $L_{v, i n s t}$. 


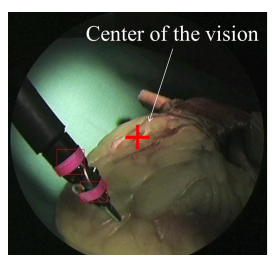

(a)

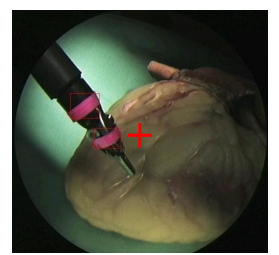

(c)

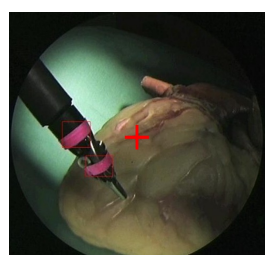

(b)

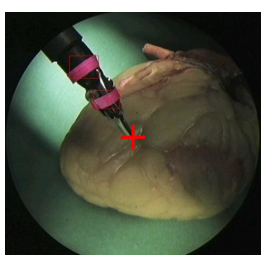

(d)
Fig. 3. Procedure of experiments for control of the laparoscope. These figures are images from the laparoscope. Positioning of the laparoscope proceeded from (a) to (d). The instrument is fixed and the laparoscope is controlled to display the instrument in the center of the image.

\section{EXPERIMENTS}

\section{A. Autonomous Positioning of Camera}

To investigate the performance of camera control, we autonomously positionined of the camera and recorded the movement of the feature point in image coordinates. The procedure of the positioning of the laparoscope is shown in Fig.3. To compare the result of this experiment, the trajectory of the feature point was simulated in the same condition. The trajectory of the feature point from the experiment and simulation are shown in Fig.4.

We have got the trajectory of the feature point as a straight line in Fig.4(a). This result is as estimated due to the fact that the interaction matrix for camera control is invertible. At the same time, the convergence of the error is also well-controlled, which is depicted in Fig.4(b). The rate of convergence is constant and the error converges to zero.

\section{B. Autonomous Positioning of Instrument}

1) Evaluation of trocar constraint: To verify the trocar constraint, we recorded the trajectory of the shaft of the instrument during the servoing. The movement of the shaft was recorded using magnetic sensors which were attached to the shaft. The visualized result is shown in Fig.5.

The result shows that the insertion point of the instrument was fixed and the error was less than $1 \mathrm{~mm}$. In laparoscopic surgery, the fulcrum at the entry point on the skin is flexible and the precision of the position of the insertion point is sufficient. Therefore, the constraint introduced in this research is verified.

2) positioning accuracy: We performed an experiment to evaluate the precision of positioning an instrument. Using the image-based visual servoing approach mentioned above, we drove the tip of a needle which was held by the surgical instrument to positions marked on a plate. The distance between the camera and the desired position was about $5 \mathrm{~cm}$.

The result of the experiment is shown in Fig.6. The errors of positioning the instrument were less than our range

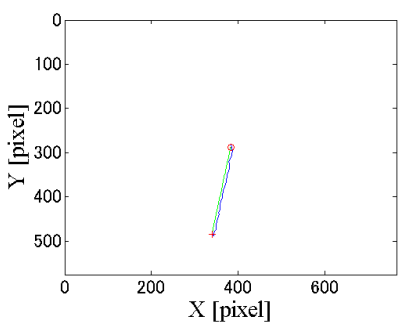

(a)

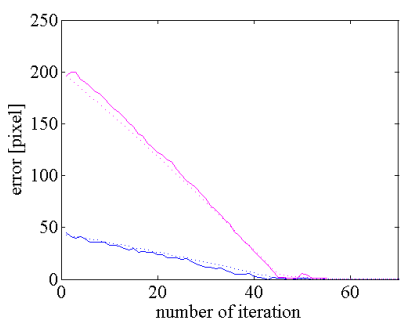

(b)

Fig. 4. Results of the simulation and the experiment for the control of the camera. Continuous lines show the result of the experiment and dot lines show the result of the simulation. (a)Trajectory of the feature point in the experiment and simulation (b)Convergence of error in the experiment and simulation

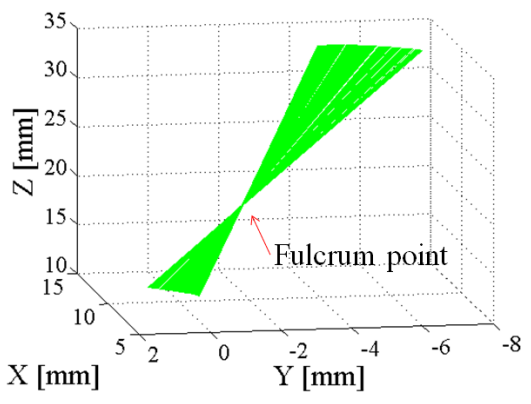

Fig. 5. Trajectory of the shaft of the instrument. This was recorded using magnetic sensors on the shaft of the instrument.

of measurement. As one can see in Fig.6, the errors of positioning the instrument were obviously less than $1 \mathrm{~mm}$ regardless of the position of the desired point in the image coordinates. The trajectory of the instrument was recorded in this experiment and the result is shown in Fig.7. Although there were small errors in tracking the surgical needles, the instrument was controlled as the simulation envisaged.

We measured the positioning accuracy at different distances between the laparoscope and the object. Fig. 8 shows the result in the case of 4 rows of $L_{v}$,inst as we have suggested above and the result in the case of 3 rows of $L_{v, \text { inst }}$. The result in the case of 4 rows of $L_{v, \text { inst }}$ shows that the positioning accuracy is very good within the range of $60 \mathrm{~mm}$ from the camera within $60 \mathrm{~mm}$ away from the laparoscope. But in the case where objects were over $80 \mathrm{~mm}$ away from the laparoscope, the positioning accuracy was not satisfactory. In contrast, the result in the case of 3 rows of $L_{v, \text { inst }}$ is not better than $1 \mathrm{~mm}$ in any case. 


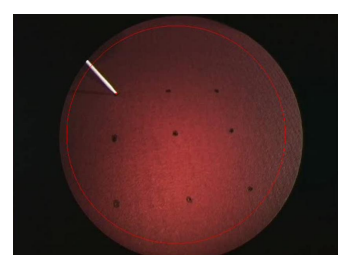

(a)

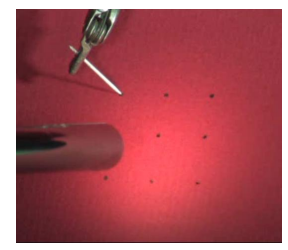

(c)

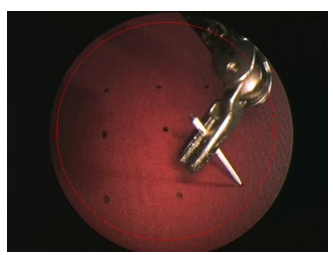

(b)

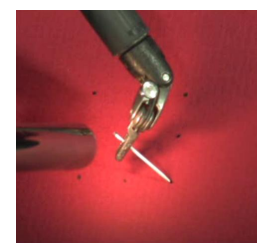

(d)
Fig. 6. Results of pointing experiments. (a) and (b) are the images from the laparoscope. (c) and (d) are the images from another camera.

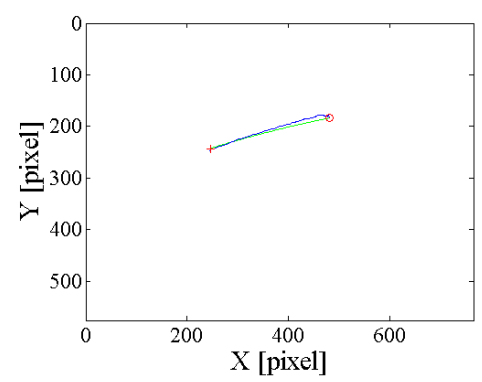

(a)

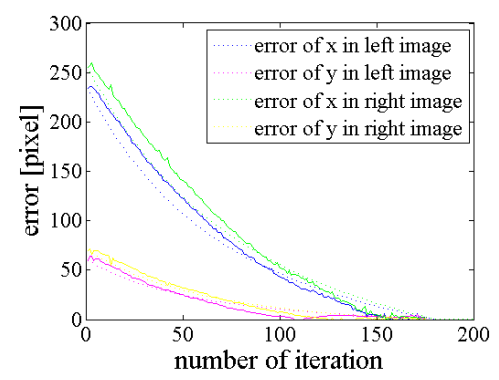

(b)

Fig. 7. Results of the simulation and the experiment for the control of the instrument. Continuous lines show the result of the experiment and dot lines show the result of the simulation. (a)Trajectory of the feature point in the experiment and the simulation (b)Convergence of error in the experiment and the simulation

\section{CONCLUSIONS AND FUTURE WORKS}

\section{A. Conclusions}

In this paper, we have described a robotic surgical system using visual servoing. With this system, we are able to steer the laparoscope and the instruments autonomously to a predefined desired position. To perform visual servoing in a suitable way for surgical operations, we applied a modified interaction matrix to control the camera. In order to analyze the performance of the proposed schemes, we investigated the stability of the control and the positioning accuracy. The benefit of this system was verified by successful experiments.

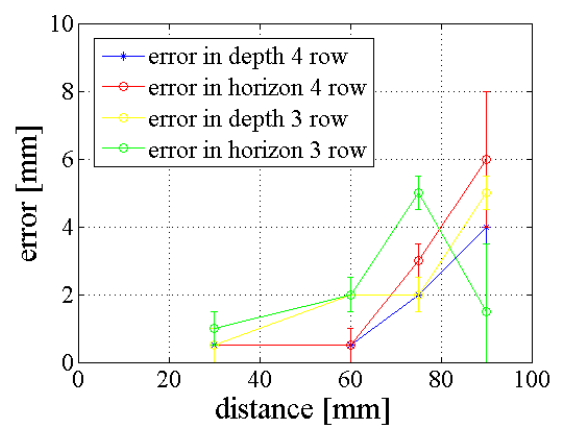

Fig. 8. Positioning accuracy. Red and blue lines show the result in case that 4 rows of $L_{v}$,inst were used. Green and Yellow lines show the result in case that 3 rows of $L_{v}$,inst were used

\section{B. Future Works}

The instrument tracking should be improved to apply this system to real surgical operations. Tracking the instruments has to be robust against occlusion, adhesion of blood to the instruments or noises in laparoscopic images. After development of an appropriate method to track the instrument, the next step is to perform more complicated tasks, such as autonomous knot-tying or stitching.

\section{REFERENCES}

[1] F. Chaumette and S. Hutchinson, "Visual servo control, part i: Basic approaches," IEEE Robotics and Automation Magazine, vol. 13, no. 4, pp. 82-90, December 2006.

[2] - "Visual servo control, part ii: Advanced approaches," IEEE Robotics and Automation Magazine, vol. 14, no. 1, pp. 109-118, March 2007.

[3] R. Ginhoux, J. Gangloff, M. de Mathelin, L. Soler, M. Sanchez, and J. Marescaux, "Active filtering of physiological motion in robotized surgery using predictive control," Robotics, IEEE Transactions on, vol. 21, no. 1, pp. 67-79, Feb. 2005.

[4] — , "Beating heart tracking in robotic surgery using $500 \mathrm{hz}$ visual servoing, model predictive control and an adaptive observer," vol. 1, April-1 May 2004, pp. 274-279 Vol.1.

[5] G. Hager, "A modular system for robust positioning using feedback from stereo vision," Robotics and Automation, IEEE Transactions on, vol. 13, no. 4, pp. 582-595, Aug 1997.

[6] P. Hynes, G. Dodds, and A. Wilkinson, "Uncalibrated visual-servoing of a dual-arm robot for surgical tasks," in Computational Intelligence in Robotics and Automation, 2005. CIRA 2005. Proceedings. 2005 IEEE International Symposium on, June 2005, pp. 151-156.

[7] — , "Uncalibrated visual-servoing of a dual-arm robot for mis suturing," in Biomedical Robotics and Biomechatronics, 2006. BioRob 2006. The First IEEE/RAS-EMBS International Conference on, Feb. 2006, pp. 420-425.

[8] D. Kragic and H. I. Christensen, "A framework for visual servoing," Computer Vision Systems, vol. Volume 2626/2003, pp. 345-354, 2003

[9] D. Kragic, D. Kragic, H. I. Christensen, and H. I. Christensen, "Survey on visual servoing for manipulation," Computational Vision and Active Perception Laboratory, Tech. Rep., 2002.

[10] J. Leven, D. Burschka, R. Kumar, M. Choti, C. Hasser, and R. H. Taylor, "Davinci canvas: A telerobotic surgical system with integrated, robot-assisted, laparoscopic ultrasound capability," Medical Image Computing and Computer-Assisted Intervention, MICCAI 2005, vol. Volume 3749/2005, pp. 811-818, 2005.

[11] H. Mayer, I. Nagy, A. Knoll, E. Schirmbeck, and R. Bauernschmitt, "Robotic system to evaluate force feedback in minimally invasive computer aided surgery," in DETC, ASME Design Engineering Technical Conferences, 2004.

[12] I. Nagy, H. Mayer, A. Knoll, E. Schirmbeck, and R. Bauernschmitt, "The endo[pa]r system for minimally invasive robotic surgery," in the IEEE/RSJ International Conference on Intelligent Robots and Systems (IROS), 2004 